\title{
Rotating multicomponent Bose-Einstein condensates
}

\author{
Zuhan Liu
}

\begin{abstract}
In the understanding of the spatial behavior of interacting components of multicomponent Bose-Einstein condensates (BECs), a central problem is to establish whether coexistence of all the components occurs, or the interspecies interaction leads to extinction, that is, configurations where one or more densities are null. In this paper, for the rotating $k$-mixture BEC, we prove that the interspecies interaction leads to extinction in the Thomas-Fermi approximation.
\end{abstract}

Mathematics Subject Classification (2000). 35B65, 35K55.

Keywords. Bose-Einstein condensate, Phase separation, Thomas-Fermi approximation.

\section{Introduction}

A Bose-Einstein condensate (BEC) is a state of matter of bosons confined in an external potential and cooled to temperatures very near to absolute zero. Under such conditions, a large fraction of the atoms collapse into the lowest quantum state of the external potential, at which point quantum effects become apparent on a macroscopic scale. This state of matter was first predicted by Satyendra Nath Bose and Albert Einstein in 1924-1925. Since the first experimental achievement of BEC in dilute bosonic atomic gases in 1995 and the Nobel Prize in 2001 [2,9,17], BECs have been produced and studied extensively in laboratory, and have afforded an intriguing glimpse into the macroscopic quantum world [34].

Attention has recently broadened to include exploration of quantized vortex states and their dynamics associated with superfluidity $[1,3,16,30,31]$, and of systems of two or more condensates [20]. These are some of key issues, in view of potential applications, in the study of quantized vortices which are well-known signature of superfluidity $[3-5,16,21]$. The first experiment involving the interactions between multiple-species BEC was performed in [32]. 
It demonstrated the possibility of producing long-lived multiple condensate systems, and the condensate wave function is dramatically affected by the presence of interspecies interactions. Recent experimental advances in exploration of systems of uniting two or more condensates, e.g. in a magnetic trap in rubidium [32], have spurred great excitement in the atomic physics community and renewed interest in studying the properties of two-component BEC $[6,24,25,39]$. In an ultracold dilute Bose gas, two different hyperfine spin states may repel each other and form segregated domains like the mixture of oil and water. Such a phenomenon is called phase separation of a binary mixture of BECs and has been investigated extensively by experimental and theoretical physicists [38].

By a mean-field approximation, the state of the BEC can be described by the wave function of the condensate to dilute systems. At temperatures $T$ much smaller than the critical temperature $T_{c}$ [34], for a k-component BEC, its wave function can be well described by the self-consistent nonlinear Schrödinger equations [10,24-26,30-38], know as coupled Gross-Pitaevskii equations $[6,38,39]$ :

$$
\left\{\begin{array}{c}
-i \hbar \frac{\partial u_{j}}{\partial t}=\frac{\hbar^{2}}{2 m} \Delta u_{j}+\Omega L u_{j}+\rho^{T F}(x) u_{j} \\
+\sum_{i=1}^{k} U_{i j}\left|u_{i}\right|^{2} u_{j} \quad \text { in } \mathcal{D} \times(0,+\infty), \\
u_{j}=0 \text { in } \partial \mathcal{D}, j=1,2, \ldots, k
\end{array}\right.
$$

$u_{j}$ denotes the macroscopic wave function of the $j$ th $(j=1,2, \ldots, k)$ component, $\left|u_{j}\right|^{2}$ is interpreted as the particle density of the $j$ th component. $\Omega$ is the angular velocity of the rotating laser beam, $L=-i\left(x_{1} \partial_{x_{2}}-\right.$ $\left.x_{2} \partial_{x_{1}}\right)=-i x^{\perp} \cdot \nabla$ is the angular momentum, $\mathcal{D}$ is a bounded domain in $\mathbb{R}^{2}$. $\hbar$ is the Planck constant divided by $2 \pi$ and $m$ is atom mass. The constant $U_{j j}(j=1,2, \ldots, k)$ is the intraspecies scattering length of the $j$ th hyperfine state and $U_{i j}(i \neq j)$ is the interspecies scattering length. As $U_{j j}<0(>0)$, the self-interaction is repulsive (attractive). As $U_{i j}>0(<0)$, the interspecies interaction is attractive (repulsive). The function $\rho^{T F}(x)$ is the magnetic trapping potential and if the harmonic potential is considered, it takes the form

$$
\rho^{T F}(x)=\rho-\left(x_{1}^{2}+\alpha x_{2}^{2}\right),
$$

with $1, \alpha$ the trapping frequencies in $x_{1}, x_{2}$ directions, respectively. Thus,

$$
\mathcal{D}=\left\{x: \rho^{T F}(x)>0\right\} .
$$

$\rho$ is determined by $\int_{\mathcal{D}} \rho^{T F}=1[3]$. Thus,

$$
\mathcal{D}=\left\{x: \rho^{T F}(x)>0\right\} .
$$

For non-rotating two-component BEC, Bao [6] presents a continuous normalized gradient flow with backward Euler finite difference discretization to compute the ground state and a time-splitting sine-pseudospectral methods to compute the dynamics; Lin and Zhang [26] study the semiclassical limits of 2-mixtures of BEC (1.1); Garcia-Ripoll and Perez-Garcia [18] study the stability and dynamics of quantized vortices; Liu [27,29] analyzes the structure of the vortices and the spatial behavior in the Thomas-Fermi approximation; Lin and Wei $[24,25]$ analyze the existence of ground states and spike solutions; 
Riboli et al. [35] and Jezek et al. [22] classify different spatial patterns of the ground states; Chui et al. [13] study quantum phase separation dynamics, the effect of trap displacements and symmetric-asymmetric transition. For solitary wave solutions of the form $u_{j}(x, t)=e^{i \lambda_{j} t} \psi_{j}(x)(j=1,2)$ and $\Omega=0$ of system (1.1), one investigates the phase separation phenomena $[10,11,33,40,37]$. Where $\psi_{1}(x)$ and $\psi_{2}(x)$ are real functions, which satisfy the elliptic system.

For rotating k-mixtures BEC, there has been few analytical results about rotating multicomponent $\mathrm{BEC}$ in the literature. Zhang et al. [39] propose an efficient and accurate method for numerical simulations and investigate the dynamics of rotating two-component BEC. Liu [28] characterize the dependence of the shape of vortex line on the trap and the rotational velocity.

As $\left|U_{i j}\right|>\sqrt{\left|U_{i i}\right|\left|U_{j j}\right|}$ and $U_{i i}<0, U_{j j}<0$, spontaneous symmetric breaking occurs, and the $j$ th component and $i$ th component are immiscible and separated in space by an interface called phase separation [38]. For this reason, we may set $U_{j j}=-\varepsilon^{-2} \frac{\hbar}{m}, U_{i j}=-\left(\varepsilon^{-2}+\beta\right) \frac{\hbar}{m}$ in the system (1.1), consider the time-independent case, and transform it into the following system

$$
\left\{\begin{array}{l}
-\triangle u_{j}^{\varepsilon}-\Omega L u_{j}+\beta(\varepsilon) \sum_{i \neq j}\left|u_{i}^{\varepsilon}\right|^{2} u_{j}^{\varepsilon}=\frac{u_{j}^{\varepsilon}}{\varepsilon^{2}}\left(\rho^{T F}(x)-\sum_{i=1}^{k}\left|u_{i}^{\varepsilon}\right|^{2}\right) \quad \text { in } \mathcal{D}, \\
u_{j}^{\varepsilon}(x) \in \mathbb{C}, j=1,2, \ldots, k, \quad u_{j}^{\varepsilon}(x)=0 \quad \text { on } \partial \mathcal{D} .
\end{array}\right.
$$

The solutions to (1.4) is the critical point of the following energy functional

$$
\begin{aligned}
G\left(u_{1}, \ldots, u_{k}\right)= & \frac{1}{2} \int_{\mathcal{D}}\left(\sum_{j=1}^{k}\left|\nabla u_{j}\right|^{2}+\frac{1}{2 \varepsilon^{2}}\left(\rho^{T F}(x)-\sum_{i=1}^{k}\left|u_{i}\right|^{2}\right)^{2}\right] \\
& \left.+\beta(\varepsilon) \sum_{i \neq j}\left|u_{i}\right|^{2}\left|u_{j}\right|^{2}-\sum_{j=1}^{k} \Omega x^{\perp} \cdot\left(i u_{j}, \nabla u_{j}\right)\right)
\end{aligned}
$$

in the space $H=\left\{u_{j} \in H^{1}(\mathcal{D} ; \mathbb{C}): u_{j}=0\right.$ on $\left.\partial \mathcal{D}, j=1, \ldots, k\right\}$.

From a rigorous mathematical point of view, the phase separation is not well understood so far for the rotating $k$-mixture BEC (1.4). The method in $[10,11,33,37,40]$ can not be applied to the this case. The main purpose of this paper is to study the spatial behavior of the minimizer of the functional (1.5). In Thomas-Fermi approximation, $\varepsilon$ is small, which will be our asymptotic regime $[14,15]$. The following is our main result concerning the minimizer of $(1.5)$.

Theorem 1.1. Let $u_{j}^{\varepsilon}, j=1,2, \ldots, k$, be a global minimizer of (1.5). Assume $\beta>0$. Then, there exists a $\left(u_{1}^{0}, u_{2}^{0}, \ldots, u_{k}^{0}\right)$ of complex-valued functions such that, up to a subsequence,

(i) $\quad u_{1}^{\varepsilon}(x) \rightarrow u_{1}^{0}, \ldots, u_{k}^{\varepsilon}(x) \rightarrow u_{k}^{0} \quad$ in $\quad C_{l o c}^{0,1}(\mathcal{D}) \quad$ as $\quad \varepsilon \rightarrow 0$;

(ii) There exists a $j_{0}$ satisfying $0<j_{0}<k$ such that

$$
u_{j_{0}}^{0} \neq 0, \quad u_{j}^{0} \equiv 0, \quad j \neq j_{0},
$$


that is, $k-1$ densities are asymptotic null;

(iii) $\quad u_{j_{0}}^{\varepsilon} \rightarrow u_{j_{0}}^{*}=\sqrt{\rho^{T F}(x)} \exp \left(i \varphi_{j_{0}}^{*}\right) \quad$ in $\quad C_{l o c}^{n}(\mathcal{D}), \forall n \in \mathbb{N}$

where $\varphi_{j_{0}}^{*}$ satisfies

$$
-\operatorname{div}\left(\rho^{T F} \nabla \varphi_{j_{0}}^{*}\right)+\frac{\Omega}{\alpha^{2}+1}\left(x_{\alpha}^{\perp} \cdot \nabla\right) \rho^{T F}=0 \quad \text { in } \quad \mathcal{D} .
$$

Remark. $\beta>0$ implies $\left|U_{12}\right|^{2}=\left|\varepsilon^{-2}+\beta\right|^{2}\left(\frac{\hbar}{m}\right)^{2}>\varepsilon^{-4}\left(\frac{\hbar}{m}\right)^{2}=\left|U_{11}\right|\left|U_{22}\right|\left(\frac{\hbar}{m}\right)^{2}$. From Theorem 1.1, one component is an asymptotic null, which means the strong interspecies interaction leads to extinction.

Due to the appearance of the angular momentum rotation term, new difficulties are introduced mathematically. Currently, there has been few analytical results about rotating multicomponent BEC in the literature. In Theorem 1.1, (1.9) describe the effect of the rotating term. In order to overcome the difficulty arising from the rotating term, we introduce the decomposition (2.3). Secondly, we introduce a map problem $\left(P^{*}\right)$ (see Sect. 3$)$. We find that the minimizer of (1.5) converges the minimizer of $\left(P^{*}\right)$ in $H^{1}$ (see Sect. 3). The third important step in our proof is to prove $\sum_{j}\left|u_{j}^{\varepsilon}\right|>0$ provided $\varepsilon$ is small. The fourth step is to prove the Bochner type inequality and small energy regularity theorem, which implies the uniformly Lipschitz estimate for $\left(u_{1}^{\varepsilon}, \ldots, u_{k}^{\varepsilon}\right)$. The fifth step is to obtain $C^{n}$-estimates by Schauder theory. Compared to [29], we improve the condition concerning $\beta$. In [29], we need the condition $\beta \rightarrow+\infty$ as $\varepsilon \rightarrow 0$.

The rest of this paper is organized as follows: In Sect. 2, we derive some basic lemmas. In Sect. 3, we prove the main Theorem 1.1.

\section{Preliminaries}

In this section, we will derive some basic lemmas. First of all, we introduce the functional, inspired from the work of Lassoued and Mironescu [23], $G_{\varepsilon}=$ $\int_{\mathcal{D}} \frac{1}{2}\left[|\nabla f|^{2}+\frac{1}{2 \varepsilon^{2}}\left(\rho^{T F}(x)-|f|^{2}\right)^{2}\right]$ in $\mathcal{H}=\left\{f \in H^{1}(\mathcal{D}, \mathbb{R}): f=0\right.$ on $\left.\partial \mathcal{D}\right\}$. Then any minimizer of $G_{\varepsilon}$ is the unique positive solution of

$$
\left\{\begin{aligned}
-\Delta f & =\frac{1}{\varepsilon^{2}} f\left(\rho^{T F}(x)-f^{2}\right) & & \text { in } \mathcal{D}, \\
f & =0 & & \text { on } \partial \mathcal{D} .
\end{aligned}\right.
$$

In fact, we have the following results.

Lemma 2.1. ([4, Proposition 2.1]) If $0<\varepsilon \ll 1$, there exists a unique classical solution $f$ of $(2.1)$, which is the unique minimizer of $G_{\varepsilon}$ in $\mathcal{H}$. In addition, for any compact subset $K \subset \mathcal{D}$, we have the following estimates:

$$
\left\|f-\sqrt{\rho^{T F}(x)}\right\|_{C^{1}(K)} \leq C_{K} \varepsilon^{2} .
$$


Let

$$
u_{j}^{\varepsilon}=f_{\varepsilon} e^{i \Omega S} v_{j}, \quad x_{\alpha}^{\perp}=\left(-\alpha^{2} x_{2}, x_{1}\right)
$$

with

$$
S=\frac{\alpha^{2}-1}{\alpha^{2}+1} x_{1} x_{2}
$$

then $v_{j}$ satisfies

$$
\begin{aligned}
-\Delta v_{j} & +\frac{2 i \Omega}{\alpha^{2}+1}\left(x_{\alpha}^{\perp} \cdot \nabla\right) v_{j}+\frac{i \Omega}{\alpha^{2}+1} \frac{v_{j}}{f_{\varepsilon}^{2}}\left(x_{\alpha}^{\perp} \cdot \nabla\right) f_{\varepsilon}^{2} \\
- & \frac{2}{f_{\varepsilon}} \nabla f_{\varepsilon} \cdot \nabla v_{j}+\beta \sum_{i \neq j} f_{\varepsilon}^{2}\left|v_{i}\right|^{2} v_{j}=\frac{f_{\varepsilon}^{2}}{\varepsilon^{2}}\left(1-\sum_{k}\left|v_{k}\right|^{2}\right) v_{j}-m v_{j} \quad \text { in } \mathcal{D},
\end{aligned}
$$

where

$$
m \triangleq-\left(\Omega^{2}|\nabla S|^{2}-2 \Omega^{2} x^{\perp} \cdot \nabla S\right)=\frac{\alpha^{2}-1}{\left(\alpha^{2}+1\right)^{2}}\left[\left(\alpha^{2}+3\right) x_{1}^{2}-\left(3 \alpha^{2}+1\right) x_{2}^{2}\right] \Omega^{2} .
$$

Note that $f_{\varepsilon} e^{i \Omega S}$ gives, in some sense, the density profile of configurations.

Set

$$
b^{\varepsilon} \triangleq 1-\frac{\varepsilon^{2}}{f_{\varepsilon}^{2}} m
$$

Then (2.4) can be rewritten as

$$
\begin{aligned}
-\Delta v_{j} & +\frac{2 i \Omega}{\alpha^{2}+1}\left(x_{\alpha}^{\perp} \cdot \nabla\right) v_{j}+\frac{i \Omega}{\alpha^{2}+1} \frac{v_{j}}{f_{\varepsilon}^{2}}\left(x_{\alpha}^{\perp} \cdot \nabla\right) f_{\varepsilon}^{2} \\
& -\frac{2}{f_{\varepsilon}} \nabla f_{\varepsilon} \cdot \nabla v_{j}+\beta \sum_{i \neq j} f_{\varepsilon}^{2}\left|v_{i}\right|^{2} v_{j} \\
= & \frac{f_{\varepsilon}^{2}}{\varepsilon^{2}}\left(b^{\varepsilon}-\sum_{k}\left|v_{k}\right|^{2}\right) v_{j} \quad \text { in } \mathcal{D}, \quad j=1,2, \ldots, k .
\end{aligned}
$$

By the maximum principles, we have the following lemma.

Lemma 2.2. ([8]) Let $v_{j}$ be the solutions to (2.6) and $K$ be any compact subset of $\mathcal{D}$. We have

$$
\left\|v_{j}\right\|_{L^{\infty}(K)} \leq 1+C \varepsilon^{2}|\ln \varepsilon|^{2}+C \frac{\varepsilon^{2}}{\operatorname{dist}(K, \partial \Omega)^{2}}, \quad\left\|\nabla v_{j}\right\|_{L^{\infty}(K)} \leq \frac{C_{K}}{\varepsilon} .
$$

Now we give the energy estimate. 
Lemma 2.3. Let $u_{j}^{\varepsilon}, j=1,2, \ldots, k$, be a global minimizer of (1.5). Then, we have

$$
\begin{gathered}
E\left(u_{1}^{\varepsilon}, \ldots, u_{k}^{\varepsilon}\right)=\frac{1}{2} \int_{\mathcal{D}}\left(\sum_{j=1}^{k}\left|\nabla u_{j}^{\varepsilon}\right|^{2}+\frac{1}{2 \varepsilon^{2}}\left(\rho^{T F}(x)-\sum_{i=1}^{k}\left|u_{i}^{\varepsilon}\right|^{2}\right)^{2}\right. \\
\left.+\beta \sum_{i \neq j}\left|u_{i}^{\varepsilon}\right|^{2}\left|u_{j}^{\varepsilon}\right|^{2}\right) \leq C .
\end{gathered}
$$

Proof. Taking test function $u_{1}^{\varepsilon}=\rho^{T F}(x), u_{i}^{\varepsilon}=0, i=2,3, \ldots, k$, we have

$$
G\left(u_{1}^{\varepsilon}, \ldots, u_{k}^{\varepsilon}\right) \leq G\left(u_{1}^{0}, \ldots, u_{k}^{0}\right) \leq C .
$$

Hence, we obtain the following inequality

$$
E\left(u_{1}^{\varepsilon}, \ldots, u_{k}^{\varepsilon}\right) \leq C+\frac{1}{4} \int_{\mathcal{D}} \sum_{j=1}^{k}\left|\nabla u_{j}^{\varepsilon}\right|^{2}+4 \Omega^{2} \int_{\mathcal{D}} \sum_{j=1}^{k}\left|u_{j}^{\varepsilon}\right|^{2} .
$$

So, we have

$$
\begin{aligned}
& \frac{1}{4} \int_{\mathcal{D}}\left(\sum_{j=1}^{k}\left|\nabla u_{j}^{\varepsilon}\right|^{2}+\frac{1}{\varepsilon^{2}}\left(\rho^{T F}-\sum_{j}^{k}\left|u_{j}^{\varepsilon}\right|^{2}\right)^{2}+\beta \sum_{i \neq j}\left|u_{i}^{\varepsilon}\right|^{2}\left|u_{j}^{\varepsilon}\right|^{2}\right) \\
& \leq C+4 \Omega^{2} \int_{\mathcal{D}} \sum_{j=1}^{k}\left|u_{j}^{\varepsilon}\right|^{2},
\end{aligned}
$$

which implies

$$
\int_{\mathcal{D}} \sum_{j}^{k}\left|u_{j}^{\varepsilon}\right|^{4} \leq C\left(1+\varepsilon^{2}\right)+C \Omega^{4} \varepsilon^{4} .
$$

Combining (2.11) with (2.12), the conclusion follows. The conclusion follows.

\section{Proof of Theorem 1.1}

In this section, we will prove Theorem 1.1. The key point is to introduce a map problem.

We define a map $W: \mathcal{D} \rightarrow \Sigma$ as follows:

$$
W=\left(w_{1}, \ldots, w_{k}\right), \quad x \in \mathcal{D},
$$

here $\Sigma \subset \mathbb{C}^{k}$ is a space,

$$
\Sigma=\left\{W=\left(w_{1}, \ldots, w_{k}\right) \in H_{0}^{1}\left(\mathcal{D}, \mathbb{C}^{3}\right): \sum_{i=1}^{k}\left|w_{i}\right|^{2}(x)=\rho^{T F}(x) \text { a.e. } x \in \mathcal{D}\right\} \text {. }
$$


We can now consider the mapping problem:

$$
\left(P^{*}\right) \quad \min \left\{\int_{\mathcal{D}} \frac{1}{2}\left(|\nabla W|^{2}-\sum_{j=1}^{k} \Omega x^{\perp} \cdot\left(i w_{j}, \nabla w_{j}\right)\right) d x: W \in \Sigma\right\} .
$$

It is rather easy to see problem $P^{*}$ has an absolute minimizer, say $W_{0}=$ $\left(w_{1}^{0}(x), \ldots, w_{k}^{0}(x)\right)$.

Lemma 3.1. Let $u_{j}^{\varepsilon}, j=1,2, \ldots, k$, be a global minimizer of (1.5). Assume $\beta>0$. Then, there exist $k$ functions $u_{1}^{0}, \ldots, u_{k}^{0} \in H^{1}(\mathcal{D}, \mathbb{C})$ such that, up to a subsequence,

$$
\begin{aligned}
& u_{1}^{\varepsilon} \rightarrow u_{1}^{0}, \ldots, u_{k}^{\varepsilon} \rightarrow u_{k}^{0} \quad \text { in } \quad H^{1}(\mathcal{D}) \quad \text { as } \quad \varepsilon \rightarrow 0 ; \\
& \beta \int_{\mathcal{D}} \sum_{i \neq j}\left|u_{i}^{\varepsilon}\right|^{2}\left|u_{j}^{\varepsilon}\right|^{2} \rightarrow 0 \quad \text { as } \quad \varepsilon \rightarrow 0 .
\end{aligned}
$$

Moreover, $W_{1}=\left(u_{1}^{0}, \ldots, u_{k}^{0}\right)$ is an absolute minimizer of the problem $P^{*}$.

Proof. By Lemma 2.3, we obtain

$$
\sum_{j}\left\|u_{j}^{\varepsilon}\right\|_{H^{1}} \leq C
$$

Hence, there exist $k$ functions $u_{1}^{0}, \ldots, u_{k}^{0} \in H^{1}(\mathcal{D}, \mathbb{C})$ such that, up to a subsequence,

$$
u_{1}^{\varepsilon} \rightarrow u_{1}^{0}, \ldots, u_{k}^{\varepsilon} \rightarrow u_{k}^{0} \quad \text { weakly in } H^{1}(\mathcal{D}) \text { and strongly in } L^{2}(\mathcal{D}) \quad \text { as } \quad \varepsilon \rightarrow 0 .
$$

Note that

$$
\int_{\mathcal{D}} \frac{\left(\rho^{T F}(x)-\sum_{j}\left|u_{j}^{\varepsilon}\right|^{2}\right)^{2}}{\varepsilon^{2}} \leq C,
$$

we obtain

$$
\sum_{j}\left|u_{j}^{0}\right|^{2}=\rho^{T F}(x) \quad \text { a.e. } \quad x \in \mathcal{D} .
$$

Hence, $W_{1}=\left(u_{1}^{0}, \ldots, u_{k}^{0}\right) \in \Sigma$. On the one hand, we obtain

$$
\begin{aligned}
\int_{\mathcal{D}}\left|\nabla W_{1}\right|^{2}-\sum_{j=1}^{k} \Omega x^{\perp} \cdot\left(i u_{j}^{0}, \nabla u_{j}^{0}\right) & \leq \underline{\lim }_{\varepsilon \rightarrow 0} \int_{\mathcal{D}} \sum_{j=1}^{k}\left|\nabla u_{j}^{\varepsilon}\right|^{2}-\sum_{j=1}^{k} \Omega x^{\perp} \cdot\left(i u_{j}^{\varepsilon}, \nabla u_{j}^{\varepsilon}\right) \\
& \leq \underline{\lim }_{\varepsilon \rightarrow 0} G\left(u_{1}^{\varepsilon}, \ldots, u_{k}^{\varepsilon}\right) \\
& \leq \int_{\mathcal{D}}\left|\nabla W_{0}\right|^{2}-\sum_{j=1}^{k} \Omega x^{\perp} \cdot\left(i w_{j}^{0}, \nabla w_{j}^{0}\right) .
\end{aligned}
$$


On the other hand, $W_{0}$ is an absolute minimizer of problem $\left(P^{*}\right)$. So, $W_{1}$ is also an absolute minimizer of problem $\left(P^{*}\right)$. Therefore, we obtain

$$
\begin{aligned}
\lim _{\varepsilon \rightarrow 0} \int_{\mathcal{D}} \sum_{j}\left|\nabla u_{j}^{\varepsilon}\right|^{2}-\sum_{j=1}^{k} \Omega x^{\perp} \cdot\left(i u_{j}^{\varepsilon}, \nabla u_{j}^{\varepsilon}\right) & =\int_{\mathcal{D}}\left|\nabla W_{1}\right|^{2}-\sum_{j=1}^{k} \Omega x^{\perp} \cdot\left(i u_{j}^{0}, \nabla u_{j}^{0}\right), \\
\lim _{\varepsilon \rightarrow 0} \beta \int_{\mathcal{D}} \sum_{i \neq j}\left|u_{i}^{\varepsilon}\right|^{2}\left|u_{j}^{\varepsilon}\right|^{2} & =0, \\
\lim _{\varepsilon \rightarrow 0} \int_{\mathcal{D}} \frac{\left(\rho^{T F}(x)-\sum_{j}\left|u_{j}^{\varepsilon}\right|^{2}\right)^{2}}{\varepsilon^{2}} & =0 .
\end{aligned}
$$

From (3.6), we obtain

$$
\lim _{\varepsilon \rightarrow 0} \int_{\mathcal{D}} \sum_{j=1}^{k} \Omega x^{\perp} \cdot\left(i u_{j}^{\varepsilon}, \nabla u_{j}^{\varepsilon}\right)=\int_{\mathcal{D}} \sum_{j=1}^{k} \Omega x^{\perp} \cdot\left(i u_{j}^{0}, \nabla u_{j}^{0}\right) .
$$

Combining (3.11) and (3.10), we have

$$
\lim _{\varepsilon \rightarrow 0} \int_{\mathcal{D}} \sum_{j}\left|\nabla u_{j}^{\varepsilon}\right|^{2}=\int_{\mathcal{D}}\left|\nabla W_{1}\right|^{2} .
$$

The conclusion of Lemma 3.1 follows.

Lemma 3.2. Assume that $\beta>0$. Then, $u_{1}^{0}, \ldots, u_{k}^{0}$ are locally Lipschitz continuous functions. Denote $\mathcal{D}_{j}=\left\{x \in \mathcal{D}:\left|u_{j}^{0}\right|>0\right\}, j=1,2, \ldots, k$. We have

$$
\left|v_{j}(x)\right|^{2} \rightarrow 1 \quad \text { in } \quad C_{l o c}^{k}\left(\mathcal{D}_{j}\right) \quad \text { as } \quad \varepsilon \rightarrow 0,
$$

and

$$
\left\|v_{j}\right\|_{C_{l o c}^{k}\left(\mathcal{D}_{j}\right)} \leq C, \quad \forall k \in \mathbb{N} .
$$

Proof. Step 1: Let $K \subset \mathcal{D}$ be any compact subset. $\sum_{j}\left|v_{j}(x)\right|^{2} \rightarrow 1$ uniformly on $K$ as $\varepsilon \rightarrow 0$.

Denote $v_{\varepsilon}=\left(v_{1}, v_{2}, \ldots, v_{k}\right)$. Let $x_{0} \in K$ and set $\alpha=\left|v_{\varepsilon}\left(x_{0}\right)\right|$. By Lemma 2.2, we have

$$
\left|v_{\varepsilon}\right| \leq \alpha+\frac{C}{\varepsilon} \rho \quad \text { if } \quad\left|x-x_{0}\right|<\rho<d=\operatorname{dist}(K, \partial \mathcal{D}) .
$$

Thus

$$
\left(1-\left|v_{\varepsilon}(x)\right|^{2}\right)^{2} \geq\left(1-\alpha-\frac{C}{\varepsilon} \rho\right)^{2} \quad \text { provided } \quad \frac{C \rho}{\varepsilon} \leq 1-\alpha .
$$

By (3.10), we obtain

$$
\varepsilon^{2} o(1)=\int_{B\left(x_{0}, \rho\right)}\left(1-\left|v_{\varepsilon}\right|^{2}\right)^{2} \geq \pi \rho^{2}\left(1-\alpha-\frac{C \rho}{\varepsilon}\right)^{2} .
$$

Let $\varepsilon$ be small such that

$$
\rho=\frac{\varepsilon(1-\alpha)}{2 C}<d .
$$


Hence

$$
\varepsilon^{2} o(1) \geq \pi \frac{\varepsilon^{2}(1-\alpha)^{2}}{4 C^{2}} \frac{(1-\alpha)^{2}}{4}
$$

and therefore

$$
(1-\alpha)^{4} \leq o(1)
$$

i.e., $\left|v_{\varepsilon}\right| \rightarrow$ uniformly on compact subsets of $\mathcal{D}$. The proof of Step 1 is completed.

Step 2: (Bochner type inequality) Let $v=\left(v_{1}, v_{2}, \ldots, v_{k}\right)$ and

$$
e(v)=\frac{1}{2}|\nabla v|^{2}+\frac{f^{2}}{4 \varepsilon^{2}}\left(b_{\varepsilon}-|v|^{2}\right)^{2}+\beta \sum_{i \neq j}\left|v_{i}\right|^{2}\left|v_{j}\right|^{2} .
$$

We have the following Bochner type inequality, for any given $\eta>0$,

$$
-\operatorname{div}\left(f^{2} \nabla e(v)\right) \leq C_{\eta}(1+e(v)) e(v)+\eta|v|^{2} \quad \text { in } \quad K
$$

Now we prove (3.21). Note that

$$
\triangle\left(\frac{1}{2}|\nabla v|^{2}\right)=\left|D^{2} v\right|^{2}+\sum_{k} v_{x_{k}} \triangle v_{x_{k}}
$$

where $\left|D^{2} v\right|^{2}=\sum_{i, j}\left|\frac{\partial^{2} v}{\partial x_{i} \partial x_{j}}\right|^{2}$. Using (2.6) we find

$$
\begin{aligned}
-\Delta v_{j x_{i}}= & \frac{2 \nabla f}{f} \nabla v_{j x_{i}}+\left(\frac{2 \nabla f}{f}\right)_{x_{i}} \nabla v_{j}-\beta \sum_{i \neq j}\left(f^{2}\left|v_{i}\right|^{2} v_{j}\right)_{x_{i}} \\
& -\frac{2 i \Omega}{\alpha^{2}+1}\left(x_{\alpha}^{\perp} \cdot \nabla\right) v_{j x_{i}}-\left(\frac{2 i \Omega}{\alpha^{2}+1}\left(x_{\alpha}^{\perp}\right)\right)_{x_{i}} \cdot \nabla v_{j} \\
& -\frac{i \Omega}{\alpha^{2}+1} \frac{1}{f_{\varepsilon}^{2}}\left(x_{\alpha}^{\perp} \cdot \nabla\right) f_{\varepsilon}^{2} v_{j x_{i}}-\left(\frac{i \Omega}{\alpha^{2}+1} \frac{1}{f_{\varepsilon}^{2}}\left(x_{\alpha}^{\perp} \cdot \nabla\right) f_{\varepsilon}^{2}\right)_{x_{i}} v_{j} \\
& +\frac{2 f^{2}}{\varepsilon^{2}}\left(b_{\varepsilon x_{i}}-v v_{x_{i}}\right) v_{j}+\frac{f f_{x_{i}}}{\varepsilon^{2}}\left(b_{\varepsilon}-|v|^{2}\right) v_{j}+\frac{f^{2}}{\varepsilon^{2}}\left(b_{\varepsilon}-|v|^{2}\right) v_{j x_{i}} .
\end{aligned}
$$


Inserting this into (3.22) and using (2.6) we see that

$$
\begin{aligned}
& -\triangle\left(\frac{1}{2}|\nabla v|^{2}\right)=-\frac{2 \nabla f}{f} \cdot \nabla\left(\frac{1}{2}|\nabla v|^{2}\right)-\left|D^{2} v\right|^{2}-\frac{f^{2}}{\varepsilon^{2}} \sum_{k}\left(v \cdot v_{x_{k}}\right)^{2} \\
& +\sum_{j, k}\left(\frac{2 \nabla f}{f}\right)_{x_{k}} \nabla v_{j} v_{j x_{k}}-\sum_{j, k}\left(\frac{m}{f_{\varepsilon}^{2}}\right)_{x_{k}} v_{j} v_{j x_{k}}-\sum_{j, k} \frac{2 i \Omega}{\alpha^{2}+1}\left(x_{\alpha}^{\perp} \cdot \nabla\right) v_{j x_{k}} v_{j x_{k}} \\
& -\sum_{j, k}\left(\frac{2 i \Omega}{\alpha^{2}+1}\left(x_{\alpha}^{\perp}\right)\right)_{x_{k}} \cdot \nabla v_{j} v_{j x_{k}}-\sum_{j, k} \frac{i \Omega}{\alpha^{2}+1} \frac{v_{j x_{k}}}{f_{\varepsilon}^{2}}\left(x_{\alpha}^{\perp} \cdot \nabla\right) f_{\varepsilon}^{2} v_{j x_{k}} \\
& -\sum_{j, k}\left(\frac{i \Omega}{\alpha^{2}+1} \frac{1}{f_{\varepsilon}^{2}}\left(x_{\alpha}^{\perp} \cdot \nabla\right) f_{\varepsilon}^{2}\right)_{x_{k}} v_{j} v_{j x_{k}}-\beta \sum_{k} \sum_{i \neq j}\left(f^{2}\left|v_{i}\right|^{2} v_{j}\right)_{x_{k}} v_{j x_{k}} \\
& +|\nabla v|^{2} \frac{f^{2}}{\varepsilon^{2}}\left(b_{\varepsilon}-|v|^{2}\right)+\sum_{k} \frac{f f_{x_{k}}}{\varepsilon^{2}}\left(b_{\varepsilon}-|v|^{2}\right) v v_{x_{k}} \leq-\frac{2 \nabla f}{f} \cdot \nabla\left(\frac{1}{2}|\nabla v|^{2}\right) \\
& -\left|D^{2} v\right|^{2}-\frac{f^{2}}{\varepsilon^{2}} \sum_{k}\left(v \cdot v_{x_{k}}\right)^{2}+\sum_{j, k}\left(\frac{2 \nabla f}{f}\right)_{x_{k}} \nabla v_{j} v_{j x_{k}}-\sum_{j, k}\left(\frac{m}{f_{\varepsilon}^{2}}\right)_{x_{k}} v_{j} v_{j x_{k}} \\
& -\sum_{j, k} \frac{2 i \Omega}{\alpha^{2}+1}\left(x_{\alpha}^{\perp} \cdot \nabla\right) v_{j x_{k}} v_{j x_{k}}-\sum_{j, k}\left(\frac{2 i \Omega}{\alpha^{2}+1}\left(x_{\alpha}^{\perp}\right)\right)_{x_{k}} \cdot \nabla v_{j} v_{j x_{k}} \\
& -\sum_{j, k} \frac{i \Omega}{\alpha^{2}+1} \frac{v_{j x_{k}}}{f_{\varepsilon}^{2}}\left(x_{\alpha}^{\perp} \cdot \nabla\right) f_{\varepsilon}^{2} v_{j x_{k}}-\sum_{j, k}\left(\frac{i \Omega}{\alpha^{2}+1} \frac{1}{f_{\varepsilon}^{2}}\left(x_{\alpha}^{\perp} \cdot \nabla\right) f_{\varepsilon}^{2}\right)_{x_{k}} v_{j} v_{j x_{k}} \\
& +C \frac{|\nabla v|^{2}+|\nabla v|}{|v|}\left(|\Delta v|+\left|\frac{2 \nabla f}{f} \nabla v\right|+\left|\frac{2 i \Omega}{\alpha^{2}+1}\left(x_{\alpha}^{\perp} \cdot \nabla\right) v_{j}\right|\right. \\
& \left.+\left|\frac{i \Omega}{\alpha^{2}+1} \frac{v_{j}}{f_{\varepsilon}^{2}}\left(x_{\alpha}^{\perp} \cdot \nabla\right) f_{\varepsilon}\right|+\beta \sum_{i \neq j} f^{2}\left|v_{i}\right|^{2}\left|v_{j}\right|\right)-\beta \sum_{k} \sum_{i \neq j}\left(f^{2}\left|v_{i}\right|^{2} v_{j}\right)_{x_{k}} v_{j x_{k}} \text {. }
\end{aligned}
$$

Since $|\triangle v| \leq C\left|D^{2} v\right|$ and $|v| \geq \frac{1}{2}$ in $K$, using the Hölder inequality, we have, for any given $\eta>0$, that

$$
\begin{aligned}
-\frac{1}{f^{2}} \operatorname{div}\left(f^{2} \nabla\left(\frac{1}{2}|\nabla v|^{2}\right)\right) \leq & -\frac{1}{2}\left|D^{2} v\right|^{2}+C_{\eta}(1+e(v)) e(v) \\
& +\frac{1}{8} \beta^{2}(\varepsilon) \sum_{i \neq j}\left|v_{i}\right|^{4}\left|v_{j}\right|^{2}+\eta|v|^{2} .
\end{aligned}
$$

Similarly, using (2.6), we have

$$
\begin{aligned}
- & \frac{1}{f^{2}} \operatorname{div}\left(f^{2} \nabla \frac{f^{2}\left(b_{\varepsilon}-|v|^{2}\right)^{2}}{\varepsilon^{2}}\right)=-\left(\triangle f^{2}\right) \frac{\left(b_{\varepsilon}-|v|^{2}\right)^{2}}{\varepsilon^{2}} \\
& -\frac{f^{2}}{\varepsilon^{2}} \sum_{k}\left(\left(\frac{m}{f_{\varepsilon}^{2}}\right)_{x_{k}}-v v_{x_{k}}\right)^{2}+|\nabla v|^{2} \frac{f^{2}}{\varepsilon^{2}}\left(b_{\varepsilon}-|v|^{2}\right)+\frac{f^{2}}{\varepsilon^{2}}\left(b_{\varepsilon}-|v|^{2}\right) v \Delta v \\
& -\frac{f^{2}}{\varepsilon^{2}}\left(b_{\varepsilon}-|v|^{2}\right) \triangle\left(\frac{m}{f_{\varepsilon}^{2}}\right)-\frac{1}{f^{2}} \nabla f^{2} \nabla \frac{f^{2}\left(b_{\varepsilon}-|v|^{2}\right)^{2}}{\varepsilon^{2}} \\
\leq & -\frac{1}{2}|\triangle v|^{2}+C_{\eta}(1+e(v)) e(v)+\frac{5}{8} \beta^{2}(\varepsilon) \sum_{i \neq j}\left|v_{i}\right|^{4}\left|v_{j}\right|^{2}+\eta|v|^{2} .
\end{aligned}
$$


Using equation (2.6), the same computing of (3.25) gives

$$
\begin{aligned}
& -\frac{1}{f^{2}} \operatorname{div}\left(f^{2} \nabla\left(\beta \sum_{i \neq j}\left|v_{i}\right|^{2}\right)\right)\left|v_{j}\right|^{2} \\
& \quad=-2 \beta \sum_{i \neq j}\left|v_{i}\right|^{2}\left|\nabla v_{j}\right|^{2}-\beta^{2}(\varepsilon) \sum_{i \neq j}\left|v_{i}\right|^{4}\left|v_{j}\right|^{2}+\frac{2 f^{2}}{\varepsilon^{2}}\left(1-|v|^{2}\right) \beta \sum_{i \neq j}\left|v_{i}\right|^{2}\left|v_{j}\right|^{2} \\
& \leq-\frac{1}{2}|\triangle v|^{2}-\frac{7}{8} \beta^{2}(\varepsilon) \sum_{i \neq j}\left|v_{i}\right|^{4}\left|v_{j}\right|^{2}+C(1+e(v)) e(v) .
\end{aligned}
$$

Combining (3.25), (3.26) with (3.27) we obtain (3.21).

Step 3: (Small energy regularity theorem) There are two positive constants $\theta_{0} \in(0,1)$ and $K_{0}$ such that

$$
\int_{B_{R}\left(x_{0}\right)} e(v) \leq \theta_{0}
$$

then

$$
(\delta R)^{2} \sup _{B_{\delta R}\left(x_{0}\right)} e(v)(x) \leq K_{0} \int_{B_{R}\left(x_{0}\right)} e(v) d x
$$

with a constant $\delta>0$, independent of $\varepsilon$. Here $B_{R}\left(x_{0}\right) \subseteq K$. We will use the method from the Lemma 2.4 in [12].

Set $r_{1}=\delta R, \delta \in(0,1 / 2)$ to be determined in the sequel. Remark that $\delta \approx|\log R|^{-1 / 2}$ for small $R$ and may be chosen independent of $R$, if $R \geq 1$. Here with this choice of $\delta$, for $z_{0} \in B_{r}\left(x_{0}\right), r+\sigma<r_{1}$ we may estimate

$$
\int_{B_{\sigma}\left(z_{0}\right)} e(v) \leq \theta_{0} .
$$

Since $v$ is regular, there exists $\sigma_{0} \in\left(0, r_{1}\right)$ such that

$$
\left(r_{1}-\sigma_{0}\right)^{2} \sup _{B_{\sigma_{0}}\left(x_{0}\right)} e(v)=\max _{0 \leq \sigma \leq r_{1}}\left(r_{1}-\sigma\right)^{2} \sup _{B_{\sigma}\left(x_{0}\right)} e(v) .
$$

Moreover, there exists $y_{0} \in \overline{B_{\sigma_{0}}\left(x_{0}\right)}$ such that

$$
\sup _{B_{\sigma_{0}}\left(x_{0}\right)} e(v)=e(v)\left(y_{0}\right)=e_{0}
$$

Set $\rho_{0}=(1 / 2)\left(r_{1}-\sigma_{0}\right)$. By choice of $\sigma_{0}, y_{0}$, we have

$$
\sup _{B_{\rho_{0}}\left(y_{0}\right)} e(v) \leq \sup _{B_{\sigma_{0}+\rho_{0}}\left(x_{0}\right)} e(v) \leq 4 e_{0}
$$

Set

$$
r_{0}=\sqrt{e_{0}} \rho_{0}, \quad w(x)==v\left(\frac{x-x_{0}}{\sqrt{e_{0}}}+y_{0}\right) .
$$

We claim $r_{0} \leq 1$. $w$ solves $(2.6)$ in $B_{r_{0}}\left(x_{0}\right)$ with $\tilde{\varepsilon}=\varepsilon \sqrt{e_{0}}, \tilde{\beta}=\beta / e_{0} ;$ moreover, $w$ satisfies

$$
e(w)\left(x_{0}\right)=1 ; \quad \sup _{B_{r_{0}}\left(x_{0}\right)} e(w) \leq 4
$$


where $e(w)=\frac{1}{2}|\nabla w|^{2}+\left(\beta / e_{0}\right)\left|w_{1}\right|^{2}\left|w_{2}\right|^{2}+\frac{f^{2}}{4 \varepsilon^{2} e_{0}}\left(1-|w|^{2}\right)^{2}$. By Step 2, taking $\eta=\theta_{0}, e(w)$ satisfies

$$
-\operatorname{div}\left(f^{2} \nabla e(w)\right) \leq C_{\theta_{0}}\left(e(w)+\theta_{0}\right) \quad \text { in } \quad B_{r_{0}}\left(x_{0}\right)
$$

with a constant $C_{\theta_{0}}$, independent of $\varepsilon$. Thus, instead of $e(w)$ we consider the function $g(x, t)=\exp \left(-C_{\theta_{0}} t\right)\left(e(w)+\theta_{0}\right)$ in $P_{r_{0}}\left(x_{0}, 0\right)=\left\{(x, t):\left|x-x_{0}\right|<r_{0}\right.$, $\left.|t|<r_{0}^{2}\right\}$. If $r_{0} \geq 1$, then, $g(x, t)$ satisfies

$$
\partial_{t} g-\operatorname{div}\left(f^{2} \nabla g\right) \leq 0 \quad \text { in } \quad P_{r_{0}}\left(x_{0}, 0\right)
$$

Moser's Harnack inequality implies the estimate

$$
1 \leq g\left(x_{0}, 0\right)=e(w)\left(x_{0}\right)+\theta_{0} \leq C \int_{P_{1}\left(x_{0}, 0\right)} g(x, t) \leq C \int_{B_{1}\left(x_{0}\right)}\left(e(w)+\theta_{0}\right) .
$$

Since $\frac{1}{\sqrt{e_{0}}}+\sigma_{0} \leq \rho_{0}+\sigma_{0}<r_{1}$, using (3.30), we have

$$
\int_{B_{1}\left(x_{0}\right)} e(w)=e_{0} \int_{B_{\frac{1}{\sqrt{e_{0}}}}} e(v) \leq C \theta_{0}
$$

and we yield a contradiction for small $\theta_{0}$. Hence, $r_{0} \leq 1$.

By choice of $\sigma_{0}$ this implies

$$
\max _{0 \leq \sigma \leq r_{1}}\left(r_{1}-\sigma\right)^{2} \sup _{B_{\sigma}\left(x_{0}\right)} e(v) \leq 4 \rho_{0}^{2} e_{0}=4 r_{0}^{2} \leq 4 .
$$

Hence, we may choose $\sigma=(1 / 2) r_{1}=(\delta / 2) R$ and divide by $\sigma^{2}$ to complete the proof.

Step 4: By Step 1, there is an $r_{0}>0$ such that

$$
\int_{B_{r_{0}}\left(x_{0}\right)}\left|\nabla W_{1}\right|^{2} \leq \theta_{0} / 2
$$

From (3.10) and (3.12), for all $\varepsilon$ small, $\int_{B_{r_{0}}\left(x_{0}\right)} e(v) \leq \theta_{0}$. Now using the small energy regularity theorem we have that

$$
e(v)(x) \leq C \theta_{0}, \quad x \in B_{r_{0} / 2}\left(x_{0}\right) .
$$

Then, using the finite covering theorem, for any compact subset $K \subset \Omega$, we have

$$
e(v) \leq C \quad \text { in } \quad K
$$

Step 5: Let $K \subseteq \Omega_{j}$ be any compact subdomain. By Lemma 3.1 and Step 4 , we have $u_{i}^{0}=0$ in $\Omega_{j}, \quad i \neq j$, and

$$
u_{i}^{\varepsilon} \rightarrow 0 \quad \text { uniformly in } K \subset \Omega_{j} \quad \text { as } \quad \varepsilon \rightarrow 0 .
$$

By Step 1, we may assume that $\varepsilon$ is sufficiently small so that

$$
\left|v_{j}^{\varepsilon}(x)\right| \geq 1 / 2 \quad \text { in } \quad K \subset \Omega_{j} .
$$

Thus we may write

$$
v_{j}^{\varepsilon}(x)=\rho_{\varepsilon}(x) \exp \left(i \varphi_{\varepsilon}(x)\right) \quad \text { in } K,
$$


and we may assume

$$
\frac{1}{|K|} \int_{K} \varphi^{\varepsilon} \in[0,2 \pi) .
$$

Using (2.6), we have

$$
\begin{aligned}
& -\operatorname{div}\left(f_{\varepsilon}^{2} \nabla \varphi_{\varepsilon}\right)+\frac{\Omega}{\alpha^{2}+1}\left(x_{\alpha}^{\perp} \cdot \nabla f_{\varepsilon}^{2}+2 f_{\varepsilon}^{2} x_{\alpha}^{\perp} \cdot \nabla \rho_{\varepsilon}\right)=0 \quad \text { in } K, \\
& -\operatorname{div}\left(f_{\varepsilon}^{2} \nabla \rho_{\varepsilon}\right)+f_{\varepsilon}^{2} \rho_{\varepsilon}\left|\nabla \varphi_{\varepsilon}\right|^{2}-\frac{2 f_{\varepsilon}^{2} \Omega}{\alpha^{2}+1}\left(x_{\varepsilon}^{\perp} \cdot \nabla\right) \varphi_{\varepsilon}+2 f_{\varepsilon}^{2} \beta \sum_{i \neq j}\left|v_{i}\right|^{2} \rho_{\varepsilon} \\
& =\frac{f_{\varepsilon}^{4}}{\varepsilon^{2}}\left(b_{\varepsilon}-\left|v_{\varepsilon}\right|^{2}\right) \rho_{\varepsilon} \quad \text { in } K .
\end{aligned}
$$

We have, for $\varepsilon<\varepsilon_{0}$ and $K_{1} \subset K$,

$$
\left\|\varphi_{\varepsilon}\right\|_{C^{1, \theta_{0}\left(K_{1}\right)}} \leq C,\left.|1-| v_{\varepsilon}\right|^{2} \mid \leq C \varepsilon^{2} \quad \text { in } \quad K_{1} .
$$

Now we prove (3.49). By Step 4, we have, for $0<\theta_{0}<1$,

$$
\left\|\rho_{\varepsilon}^{2}\right\|_{C^{0, \theta_{0}(K)}} \leq C .
$$

Using Schauder theory [19], it follows that

$$
\left\|\varphi_{\varepsilon}\right\|_{C^{1, \theta_{0}\left(K_{1}\right)}} \leq C\left(\left\|\varphi_{\varepsilon}\right\|_{C^{0, \theta_{0}(K)}}+\left\|\rho_{\varepsilon}\right\|_{C^{0, \theta_{0}(K)}}\right) \leq C,
$$

by Step 4 .

In order to prove second estimate, we need the following elliptic estimate

Lemma 3.3. (Lemma 2; [7]) Let $\omega(r)$ be the solution of

$$
-\varepsilon^{2} \triangle \omega+\omega=0 \quad \text { on } B(0, R), \quad \omega=1 \quad \text { on } \quad \partial B(0, R) .
$$

Then, for $\varepsilon<\frac{3}{4} R$,

$$
\omega(r) \leq e^{\frac{1}{4 \varepsilon R}\left(r^{2}-R^{2}\right)} \quad \text { on } \quad B(0, R) .
$$

Set $\xi_{\varepsilon}=1-|v|^{2}$, using (2.6), we have

$$
\begin{aligned}
\triangle \xi_{\varepsilon} & -\frac{f^{2}}{\varepsilon^{2}}|v|^{2} \xi_{\varepsilon}+2 \sum_{j}\left|\nabla v_{j}\right|^{2}+2 \beta \sum_{i \neq j}\left|v_{i}\right|^{2} \rho_{\varepsilon}^{2} \\
& +2 \frac{\Omega|v|^{2}}{\left(\alpha^{2}+1\right) f_{\varepsilon}^{2}}\left(x_{\alpha}^{\perp} \cdot \nabla\right) f_{\varepsilon}^{2}-4 \sum_{j} \frac{\Omega}{\alpha^{2}+1} x_{\alpha}^{\perp} \cdot j\left(v_{j}\right)=\frac{2 \nabla f}{f} \nabla|v|^{2} \text { in } K .
\end{aligned}
$$

Let $d=\operatorname{dist}(K, \partial \Omega)$. Assume $x_{0}=0 \in K$. For $\varepsilon$ sufficiently small we have

$$
\left|v_{\varepsilon}\right| \geq 1 / 2 \quad \text { on } \quad B(0, d / 2)
$$

Using Step 4,

$$
-\triangle \xi_{\varepsilon}+\frac{1}{\varepsilon^{2}} \xi_{\varepsilon} \leq C \text { in } B(0, d / 2) .
$$

Using Lemma 3.3 and the maximum principle we have

$$
\xi_{\varepsilon} \leq C \varepsilon^{2}+e^{\frac{1}{2 \varepsilon d}\left(|x|^{2}-\frac{d^{2}}{4}\right)} .
$$


In particular

$$
\xi_{\varepsilon}(0) \leq C \varepsilon^{2}+\varepsilon^{2} e^{-\frac{d}{8 \varepsilon}} .
$$

This prove (3.49) since the right-hand side in (3.56) remains bounded as $\varepsilon \rightarrow 0$.

Denote

$$
\begin{aligned}
q_{\varepsilon} \equiv & \frac{f^{4}}{\varepsilon^{2}} \rho_{\varepsilon}^{2}\left(|v|^{2}-b_{\varepsilon}\right)+f^{2} \rho_{\varepsilon}^{2}\left|\nabla \varphi_{\varepsilon}\right|^{2}+2 f^{2} \beta \sum_{i \neq j}\left|v_{i}\right|^{2} \rho_{\varepsilon}^{2}+f^{2}\left|\nabla \rho_{\varepsilon}\right|^{2} \\
& +2 \frac{\Omega|v|^{2}}{\left(\alpha^{2}+1\right) f_{\varepsilon}^{2}}\left(x_{\alpha}^{\perp} \cdot \nabla\right) f_{\varepsilon}^{2}-4 \sum_{j} \frac{\Omega}{\alpha^{2}+1} x_{\alpha}^{\perp} \cdot j\left(v_{j}\right),
\end{aligned}
$$

then, from (3.43), (3.45), (3.49), we have $\left|q_{\varepsilon}\right| \leq C$ in $K_{1}$, and

$$
\operatorname{div}\left(f^{2} \nabla \rho_{\varepsilon}^{2}\right)=q_{\varepsilon} \text { in } K .
$$

Using Schauder theory [19], it follows that

$$
\left\|\rho_{\varepsilon}^{2}\right\|_{C^{1, \theta_{0}\left(K_{1}\right)}} \leq C .
$$

By the bootstrap argument we have

$$
\begin{gathered}
\left\|\varphi_{\varepsilon}\right\|_{C_{l o c}^{k}\left(\Omega_{j}\right)} \leq C, \\
\left\|\rho_{\varepsilon}\right\|_{C_{l o c}^{k}\left(\Omega_{j}\right)} \leq C .
\end{gathered}
$$

From the proof of Lemma 3.2, we have the following results.

Lemma 3.4. Under the assumption of Lemma 3.2, we have, for some sequence $\varepsilon \rightarrow 0$,

$$
v_{j}^{\varepsilon} \rightarrow v_{j}^{*}=\exp \left(i \varphi_{j}^{*}\right) \quad \text { in } \quad C_{l o c}^{k}\left(\Omega_{j}\right)
$$

where $\varphi_{j}^{*}$ satisfies

$$
-\operatorname{div}\left(\rho^{T F} \nabla \varphi_{j_{0}}^{*}\right)+\frac{\Omega}{\alpha^{2}+1}\left(x_{\alpha}^{\perp} \cdot \nabla\right) \rho^{T F}=0 \quad \text { in } \quad \Omega_{j} .
$$

Lemma 3.5. Under the assumption of Lemma 3.2, there exists a $j_{0}$ satisfying $0<j_{0}<k$ such that

$$
u_{j_{0}}^{0} \neq 0, \quad u_{j}^{0} \equiv 0, \quad j \neq j_{0},
$$

that is, $k-1$ densities are asymptotic null.

Proof. By (3.10) and (3.43), we have $\sum_{j}\left|u_{j}^{0}\right|^{2}=\rho^{T F}(x), \sum_{i \neq j}\left|u_{i}^{0}\right|^{2}\left|u_{j}^{0}\right|^{2}=0$, and, $u_{1}^{0}, \ldots, u_{k}^{0}$ are continuous. Hence, there exists a $j_{0}$ satisfying $0<j_{0}<k$ such that

$$
u_{j_{0}}^{0} \neq 0, \quad u_{j}^{0} \equiv 0, \quad j \neq j_{0},
$$

that is, $k-1$ densities are asymptotic null.

Combining Lemmas 3.1-3.5 we complete the proof of Theorem 1.1. 


\section{Acknowledgments}

This work is supported by the National Natural Science Foundation of China (No.11071206).

\section{References}

[1] Abo-Shaeer, J.R., Raman, C., Vogels, J.M., Ketterle, W.: Observation of vortes lattices in Bose-Einstein condensate. Science 292, 476-479 (2001)

[2] Anderson, M.H., Ensher, J.R., Matthews, M.R., Wieman, C.E., Cornell, E.A.: Observation of Bose-Einstein condensation in a dilution atomic vapor. Science 269, 198-201 (1995)

[3] Aftalion, A.: Vortices in Bose-Einstein Condensates, Progress in Nonlinear Differential Equations and Their Applications 67. Birkhäuser, Basel (2008)

[4] Aftalion, A., Alama, S., Bronsard, L.: Giant vortex and the breakdown of strong pinning in a rotating Bose-Einstein condensate. Arch. Ration. Mech. Anal. 178, 247-286 (2005)

[5] Aftalion, A., Du, Q.: Vortices in a rotating Bose-Einstein condensate: critical angular velocities and energy diagrams in the Thomas-Fermi regime. Phys. Rev. A 64, 063603 (2001)

[6] Bao, W.: Ground states and dynamics of multicomponent Bose-Einstein condensates. Multiscale Model. Simul. 2, 210-236 (2004)

[7] Bethuel, F., Brezis, H., Helèin, F.: Asymptotics for the minimizers of a Ginzburg-Landau functional. Calc. Var. PDE 1, 123-148 (1993)

[8] Bethuel, F., Orlandi, G., Smets, D.: Vortex rings for the Gross-Pitaevskii equation. J. Eur. Math. Soc. 6, 17-94 (2004)

[9] Bradley, C.C., Sackett, C.A., Hulet, R.G.: Bose-Einstein condensation of lithium: observation of limited condensate number. Phys. Rev. Lett. 78, 985-989 (1997)

[10] Chang, S.M., Lin, C.S., Lin, T.C., Lin, W.W.: Segregated nodal domains of two-dimensional multispecies Bose-Einstein condensates. Physica D 196, 341-361 (2004)

[11] Chang, S.M., Lin, W.W., Shieh, S.F.: Gauss-Seidel-type methods for energy states of a multi-component Bose-Einstein condensate. J. Comput. Phys. 202, 367-390 (2005)

[12] Chen, Y.M., Struwe, M.: Existence and partial regularity results for the heat flow for harmonic maps. Math. Z. 201, 83-103 (1989)

[13] Chui, S.T., Ryzhov, V.N., Tateyeva, E.E.: Phase separation and vortex states in the binary mixture of Bose-Einstein condensates. J. Exp. Theor. Phys. 91, 11831189 (2000) 
[14] Correggi, M., Rindler-Daller, T., Yngvason, J.: Rapidly rotating Bose-Einstein condensates in homogeneous traps. J. Math. Phys. 48, 102103 (2007)

[15] Correggi, M., Rindler-Daller, T., Yngvason, J.: Rapidly rotating Bose-Einstein condensates in strongly anharmonic traps. J. Math. Phys. 48, 042104 (2007)

[16] Correggi, M., Yngvason, J.: Energy and vorticity in fast rotating Bose-Einstein condensates. J. Phys. A: Math. Theor. 41, 445002 (2008)

[17] Davis, K.B., Mewes, M.O., Andrews, M.R., van Druten, N.J., Durfee, D.S., Kurn, D.M., Ketterle, W.: Bose-Einstein condensation in a gas of sodium atoms. Phys. Rev. Lett. 75, 3969-3973 (1995)

[18] Garcia-Ripoll, J.J., Perez-Garcia, V.M.: Stable and unstable vortices in multicomponent Bose-Einstein condensates. Phys. Rev. Lett. 84, 4264-4267 (2002)

[19] Gilbarg, D., Trudinger, N.S.: Elliptic partial differential equations of second order, 2nd edn. Grundlehren der Mathematischen Wissenschaften [Fundamental Principles of Mathematical Sciences], vol. 224. Springer, Berlin (1983)

[20] Hall, D.S., Matthews, M.R., Ensher, J.R., Wieman, C.E., Cornell, E.A.: Dynamics of component reparation in a binary mixture of Bose-Einstein condensates. Phys. Rev. Lett. 81, 1539-1542 (1998)

[21] Ignat, R., Millot, V.: The critical velocity for vortex existence in a twodimensional rotating Bose-Einstein condensate. J. Funct. Anal. 233, 260$306(2006)$

[22] Jezek, D.M., Capuzzi, P., Cataldo, H.M.: Structure of vortices in two-component Bose-Einstein condensates. Phys. Rev. A 64, 023605 (2001)

[23] Lassoued, L., Mironescu, P.: Ginzburg-Landau type energy with discontinuous constraint. J. Anal. Math. 77, 27-58 (1999)

[24] Lin, T.C., Wei, J.: Spikes in two coupled nonlinear Schrödinger equations. Ann. I. H. Poincarè Analyse. Non. 22, 403-439 (2005)

[25] Lin, T.C., Wei, J.: Ground state of N coupled nonlinear Schrödinger equations in $\mathbb{R}^{n}, n \leq 3$. Commun. Math. Phys. 255, 629-653 (2005)

[26] Lin, T.C., Zhang, P.: Incompressible and compressible limits of Coupled systems of nonlinear Schrödinger equations. Commun. Math. Phys. 266, 547-569 (2006)

[27] Liu, Z.: Two-component Bose-Einstein condensates. J. Math. Anal. Appl. 348, 274-285 (2008)

[28] Liu, Z.: Rotating Two-component Bose-Einstein condensates. Acta Appl. Math. 110, 367-398 (2010)

[29] Liu, Z.: Phase separation of two-component Bose-Einstein condensates. J. Math. Phys. 50, 102104 (2009)

[30] Madison, K., Chevy, F., Dalibard, J., Wohlleben, W.: Vortex formation in a stirred Bose-Einstein condensate. Phys. Rev. Lett. 84 (2000) 
[31] Madison, K., Chevy, F., Dalibard, J., Wohlleben, W.: Vortices in a stirred BoseEinstein condensate. J. Mod. Opt. 47, 1-10 (2000)

[32] Myatt, C.J., Burt, E.A., Ghrist, R.W., Cornell, E.A., Wieman, C.E.: Production of two overlapping Bose-Einstein condensates by sympathetic cooling. Phys. Rev. Lett. 78, 586-589 (1997)

[33] Noris, B., Tavares, H., Terracini, S., Verzini, G.: Uniform Hölder bound for nonlinear Schrödinger systems with strong competition. Comm. Pure Appl. Math. 63, 267-302 (2010). arXiv:0810.5537v1

[34] Pitaevskii, L.P., Stringari, S.: Bose-Einstein Condensation. Clarendon Press (2003)

[35] Riboli, F., Modugno, M.: Topology of the ground state of two interacting BoseEinstein condensates. Phys. Rev. A 65, 063614 (2002)

[36] Son, D.T., Stephanov, M.A.: Domain walls of relative phase in two-component Bose-Einstein condensates. Phys. Rev. A 65, 063621 (2002)

[37] Terracini, S., Verzini, G.: Multipulse phases in k-mixtures of Bose-Einstein condensates. Arch. Ration. Mech. Anal. 194, 717-741 (2009)

[38] Timmermans, E.: Phase separation of Bose-Einstein condensates. Phys. Rev. Lett. 81, 5718-5721 (1998)

[39] Zhang, Y., Bao, W., Li, H.: Dynamics of rotating two-component Bose-Einstein condensates and its efficient computation. Physica D 234, 49-69 (2007)

[40] Wei, J., Weth, T.: Asymptotic behaviour of solutions of planar elliptic systems with strong competition. Nonlinearity 21, 305-317 (2008)

Zuhan Liu

Department of Mathematics

Xuzhou Normal University

Xuzhou 221116

China

e-mail: zuhanl@yahoo.com

Received: 27 January 2011.

Accepted: 5 May 2011. 